\title{
AVALIAÇÃO TOMOGRÁFICA DOS PEDÍCULOS VERTEBRAIS NO TRATAMENTO CIRÚRGICO DOS PACIENTES COM ESCOLIOSE IDIOPÁTICA DO ADOLESCENTE
}

\author{
COMPUTED TOMOGRAPHY SCAN EVALUATION OF VERTEBRAL PEDICLES FOR SURGICAL \\ TREATMENT OF ADOLESCENT IDIOPATHIC SCOLIOSIS
}

\section{EVALUACIÓN DE TOMOGRAFÍA COMPUTARIZADA DE PEDÍCULOS VERTEBRALES NEL TRATAMIENTO QUIRÚRGICO DE ESCOLIOSIS IDIOPÁTICA DEL ADOLESCENTE}

Alceu Gomes Chueire ${ }^{1}$, Fabiano Nogueira², Allan Hiroshi de Araujo Ono ${ }^{3}$, Mauro dos Santos Volpi ${ }^{4}$, Alceu José Fornari Chueire $^{3}$

\begin{abstract}
RESUMO
Objetivo: Determinar através de cortes tomográficos e reconstrução tridimensional a incidência de mal posicionamento de parafusos em pacientes submetidos ao tratamento cirúrgico da Escoliose idiopática do adolescente. Métodos: Foram analisados exames tomográficos de 8 pacientes, tratados cirurgicamente no Hospital de Base de São José do Rio Preto-SP, realizada instrumentação posterior partindo de T2 /T4 a L4/L5 totalizando 164 parafusos. Resultados: 32,9\% $(n=54)$ apresentavam posicionamento com risco potencial,ou seja desvio acima de 2 milímetros, sendo 20,1\% $(n=33)$ com invasão lateral, 9,1\% $(n=15)$ com invasão medial, 3,6 \%( $n=6)$ com invasão anterior. Dos parafusos que ofereciam risco potencial a relação com aspecto da curva foi de $46 \%(n=25)$ na concavidade, 35\% ( $n=19)$ na convexidade e 19\% $(n=10)$ em vértebras adjacentes a curva. Conclusão: Os limites de penetração aceitáveis, assim como os métodos de mensuração ainda não foram padronizados, a técnica "free hand' mostrou-se segura, apesar da violação dos pedículos. A tomografia computadorizada pré-operatória, auxilia no planejamento cirúrgico e na redução das complicações.
\end{abstract}

Descritores: Escoliose, Parafusos Ósseos, Fusão Vertebral.

\begin{abstract}
Objective: To Evaluate by CT scan tridimensional reconstruction, malpositioning of pedicle screws in adolescent idiopathic scoliosis patients. Methods: It was analyzed CT scan cuts of 8 patients submitted to surgical treatment in Hospital de Base de São José do Rio Preto- SP, which were performed posterior spinal fusion from T2/T4 to L4/L5, 164 screws were inserted. Results: $32,9 \%$ ( $n=54)$ were malpositioned offering potential risk, which was considered $2 \mathrm{~mm}$ deviation, 20,1\% $(n=33)$ lateral invasion, 9,1\% $(n=15)$ medial invasion, 3,6\% $(n=6)$ anterior invasion. From those malpositioned screws 46\% $(n=25)$ were in concavity, 35\% $(n=19)$ in convexity and 19\% ( $n=10)$ were nearby curve. Conclusion: Acceptable invasion boundaries were not well determined, and also measurements methods are not standardized. The "free hand" technique proved safe, besides pedicle violation. Pre-Op CT Scan showed helpful in surgery planning and avoiding complications.
\end{abstract}

Keywords: Scoliosis, Bone Screws, Spinal Fusion.

\section{RESUMEN}

Objetivo: Evaluar mediante tomografía computarizada tridimensional de reconstrucción, una mala posición de los tornillos de pedículo en pacientes con escoliosis idiopática del adolescente. Métodos: Se analizó cortes de tomografía computarizada de 8 pacientes sometidos a tratamiento quirúrgico en el Hospital de Base de São José do Rio Preto-SP, que se llevaron a cabo la fusión vertebral posterior de T2/T4 a L4/L5, 164 tornillos se insertaron. Resultados: 32,9\% ( $n=54)$ fueron mal colocados que ofrecen riesgo potencial, que se consideraba una desviación de $2 \mathrm{~mm}, 20,1 \%(n=33)$, la invasión lateral, el 9,1\% $(n=15)$ invasión medial, 3,6 \% ( $n=6)$ la invasión anterior. De los tornillos mal colocados el $46 \%(n=25)$ fueron en la concavidad, el 35\% $(n=19)$ en la convexidad y el 19\% ( $n=10)$ se curva cerca. Conclusión: Los límites aceptables invasión no se determinaron también, y también los métodos de medición no están estandarizados. La técnica "free hand" resultó segura, además de violación pedículo. Pre-Op CT Scan mostró útil en la cirugía de la planificación y evitar complicaciones.

Descriptores: Escoliosis, Tornillos Óseos, Fusión Espinal.

\section{INTRODUÇÃO}

Escoliose idiopática do adolescente é uma deformidade tridimensional da coluna vertebral com curvatura lateral maior que 10 graus associado a rotação dos corpos vertebrais sendo sua manifestação entre 10 e 18 anos. Geralmente assintomática, não causa dor ou alterações neurológicas. Porém apresenta grande impacto no aspecto físico e relações sociais ${ }^{1}$. É a deformidade mais comum da coluna vertebral, acometendo aproximadamente 0,5\% a 3\% da população na

\footnotetext{
1. Livre Docente, Chefe de disciplina Ortopedia eTraumatologia - FAMERP.

2. Médico Assistente do Departamento Neurocirurgia - FAMERP.

3. Médico Residente de Ortopedia eTraumatologia - FAMERP.

4. Mestre Doutor, Chefe da Disciplina de Ortopedia e Traumatologia - UNESP

Trabalho Realizado no Hospital de Base de São José do Rio Preto -São Paulo - Brasil

Correspondência : allanmed36@yahoo.com.br
} 
idade escolar ${ }^{2}$, ocorre em ambos os sexos, com prevalência maior no sexo feminino na proporção de 1:2 nas curvas menores ${ }^{3,16}$ e nas curvas maiores chegando a 1:8 naquelas que necessitam de tratamento cirúrgico ${ }^{4}$.

O diagnóstico é clínico e radiográfico, sendo fundamental o estudo individualizado das curvas pelo método de Cobb, imprescindível no acompanhamento da progressão da doença e escolha do método de tratamento ${ }^{5}$

O uso de parafusos pediculares no tratamento cirúrgico se tornou a técnica mais difundida atualmente, atingindo resultados superiores em termos de fixação e correção das deformidades ${ }^{6}$.

Essa técnica apresenta uma incidência de mal posicionamento quando avaliadas por tomografia pós-operatória de 9,8 a 43\%. As dificuldades técnicas na sua inserção em deformidades escolióticas, oferecem risco de lesão neurológica, vascular e visceral ${ }^{7-9}$. Essas complicações são maiores nas escolioses, devido à modificação dos pontos anatômicos de entrada, causada pela rotação vertebral e alterações morfológicas dos pedículos ${ }^{10}$. A inserção correta de parafusos pediculares depende da confecção de um ponto de entrada preciso ${ }^{11}$.

O presente estudo tem o objetivo de determinar com cortes tomográficos e reconstrução tridimensional da coluna vertebral, a incidência de mal posicionamento de parafusos em pacientes submetidos ao tratamento cirúrgico da escoliose idiopática do adolescente, além de tentar correlacionar possíveis fatores de risco assim como complicações potenciais.

\section{Casuística e Métodos}

No presente estudo 8 pacientes portadores de escoliose idiopática do adolescente com curvas maiores que 40 graus pelo método e Cobb, foram submetidos ao tratamento cirúrgico pela mesma técnica. No período de Dezembro de 2009 a Dezembro de 2010 no Hospital de Base de São José do Rio Preto - São Paulo, pela equipe de Cirurgia da Coluna. A idade variou de 12 a 18 anos, sendo 1 paciente do sexo masculino e 7 do sexo feminino, com a maior curva variando de 50 a 70 graus. Realizou-se a instrumentação posterior com parafusos pedículares de titânio e duas hastes moldadas no ato operatório, com artrodese partindo de T2 /T4 a L4/L5, totalizando a colocação de 164 parafusos.

Todos pacientes foram submetidos à tomografia computadorizada (TC) pré-operatória com objetivo de estudar os pedículos e auxiliar assim na escolha de colocação dos parafusos em determinados corpos vertebrais, evitando aqueles com alterações morfológicas mais complexas. Após o ato cirúrgico foi realizada uma nova TC com reconstrução tridimensional, digital. As imagens digitalizadas foram analisadas em todos os planos no software VEPRO -EMR Manager $\AA$ utilizando ferramenta de aumento de até $371 \%$, e medidores gráficos.

Foram considerados como aceitáveis parafusos os quais se encontravam posicionados inteiramente dentro do pedículo. Aqueles que atravessam a cortical foram mensurados nos cortes transversais, invasões maiores ou iguais a $2 \mathrm{~mm}$ foram classificadas como risco potencial:

a) Risco lateral ,ou seja, potencial de lesão à pleura e vísceras (Figura 1A); b) Risco medial, ao canal medular (Figura 1B);

c) Risco anterior, vascular (Figura 1C). Os parafusos considerados como risco potencial, foram correlacionados ao nível da vértebra, concavidade ou convexidade da curva, largura do pedículo e ao comprimento ântero-posterior da vértebra;

d) Os parafusos colocados pela técnica in-out-in (Figura 1D), não foram qualificados como risco potencial ${ }^{12}$

\section{Técnica Cirúrgica}

Paciente em decúbito ventral sobre almofadas posicionadoras, via de acesso posterior, dissecção subperiosteal das articulações facetárias, garantindo boa visualização dos pontos de entrada. Realizado orifício de entrada com punção por iniciador, perfuração com fresa quadrada manual, utilizado um "probe" de ponta esférica para verificar integridade das paredes dos pedículos selecionados. Se percebido quaisquer ruptura da integridade das paredes, um
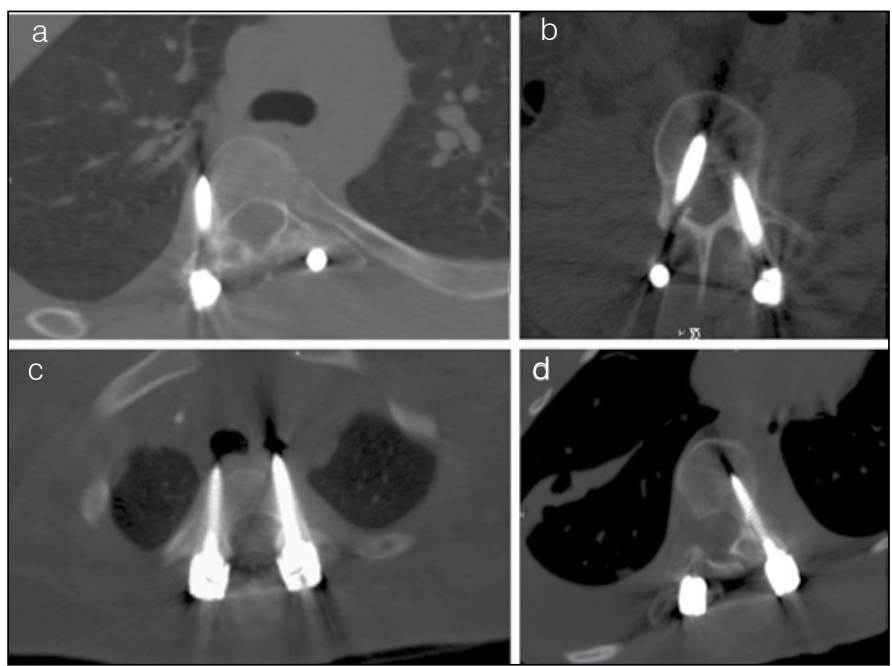

Figura 1. A, B, C E D - Risco potencial.

novo ponto de entrada numa direção diferente deveria ser confeccionado, segue-se os mesmos passos. Em seguida a colocação de parafusos pediculares de titânio e seu posicionamento foram confirmados por fluoroscopia. A correção da deformidade foi realizada pela introdução de duas barras longitudinais moldadas previamente pelo cirurgião com a preocupação da correção no plano sagital, utilizou-se 2 cross-links para aumento da estabilidade, foi realizado decorticação das facetas articulares e enxerto ósseo autólogo retirado dos processos espinhosos e do osso ilíaco.

Utilizou-se teste de Stagnara (teste do despertar) como ferramenta de avaliação neurológica intraoperatória ${ }^{15}$

\section{RESULTADOS}

Dos parafusos colocados, 32,9\% $(n=54)$ apresentavam posicionamento com risco potencial, desvio acima de 2 milímetros, sendo 20,1\% ( $n=33)$ com invasão lateral, 9,1\% $(n=15)$ com invasão medial, 3,6 \%(n=6) com invasão anterior. Os parafusos com risco potencial apresentaram a seguinte distribuição em relação ao nível vertebral em que se encontravam (Figura 2).

Dos parafusos que ofereciam risco potencial a relação com aspecto da curva foi de $46 \%(n=25)$ na concavidade, $35 \%(n=19)$ na convexidade e 19\% $(n=10)$ em vértebras adjacentes a curva.

A Tabela 1 traz um resumo das características básicas de cada paciente, classificação, idade, sexo, amplitude das curvas, angulação e o índice de mal posicionamento.

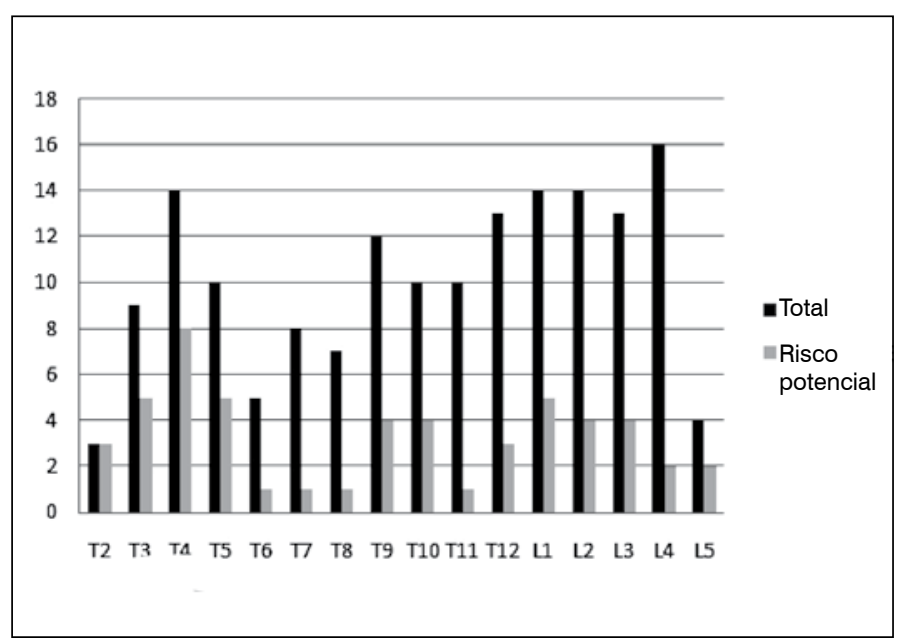

Figura 2. Distribuição em relação ao nível vertebral. 
Tabela 1. Características básicas de cada paciente.

\begin{tabular}{|c|c|c|c|c|c|c|c|c|c|c|c|}
\hline Iniciais & Sexo & Idade & Curva principal & Curvas & Cobb prévio & Sem risco & Risco potencial & Lateral & Medial & Anterior & \\
\hline 1 & FEM & 17 & Dupla principal & T6-T11/T11-L4 & $55^{\circ} / 40^{\circ}$ & 12 & 10 & 9 & 1 & 0 & 22 \\
\hline 2 & FEM & 16 & Dupla principal & T4-T11/T11-L4 & $60^{\circ} / 40^{\circ}$ & 10 & 8 & 5 & 3 & 0 & 18 \\
\hline 4 & MASC & 17 & Dupla principal & T4-T11/T12-L5 & $50^{\circ} / 55^{\circ}$ & 15 & 3 & 3 & 0 & 0 & 18 \\
\hline 5 & FEM & 18 & Toracica principal & T4-T11/T11-L1 & $64^{\circ} / 52^{\circ}$ & 20 & 6 & 3 & 3 & 0 & 26 \\
\hline 8 & FEM & 14 & Dupla principal & T5-T11/T11-L4 & $52^{\circ} / 50^{\circ}$ & 14 & 9 & 5 & 4 & 0 & 23 \\
\hline Total & & & & & & 110 & 54 & 33 & 15 & 6 & 164 \\
\hline
\end{tabular}

\section{DISCUSSÃO}

Modi et. al classificou os parafusos desviados em grau I < $2 \mathrm{mms}$, grau II entre $2 \mathrm{~mm}$ e $4 \mathrm{~mm}$, grau III entre $4,1 \mathrm{~mm}$ e $6 \mathrm{~mm}$, e grau IV entre 6,1 e 8mm; os graus III e IV foram considerados como risco potencial". Sarlak et al. Considerou como "inaceitáveis" parafusos com desvios laterais maiores que $6 \mathrm{~mm}$ e mediais maiores que $2 \mathrm{~mm}^{8}$. Ambos estudos apresentaram respectivamente $31,3 \%$ e $29 \%$ de parafusos mal posicionados (desvios maiores que $2 \mathrm{~mm}$ ) sendo 10,3\% e 10,8\% com invasão medial; $21 \%$ e 18,3\% lateral e $3,6 \%$ e $0 \%$ anterior, semelhantes aos dados encontrados no presente estudo.

Em nossa casuística: 10,2\%( $n=12)$ parafusos considerados como grau III e 7,6\%(n=9) como grau IV de Modi et al. ou "inaceitáveis".

Ebrehein et al. Assim como Polly et al. sugerem que desvios mediais $\leq 2 \mathrm{~mm}$ e laterais $\geq 6 \mathrm{~mm}$ possuem poucas ou nenhuma repercussões clínicas e anatômicas ${ }^{17,18}$

Encontramos no presente estudo diferenças significativas na colocação dos parafusos da concavidade. Wang et al. e Samdani et al. não encontram diferenças significantes entre a convexidade e concavidade na acurácia da colocação de parafusos ${ }^{9,19}$.

Métodos de imagem estão sendo desenvolvidos para auxílio intraoperatório, porém poucos são os disponíveis na maioria dos serviços. As demais séries relatam que estes tornam os procedimentos mais longos e de custo muito elevado ${ }^{14}$.

O planejamento pré-operatório com auxílio da TC assim como a reconstrução tridimensional, permitem a identificação de segmentos onde os pedículos apresentam maiores alterações morfológicas possibilitando evitá-los durante o ato cirúrgico ${ }^{13,20}$. Essa preocu-

\section{REFERENCIAS}

1. Scoliosis Research Society; Disponivel em www.srs.org

2. Rocha LE, Santili C, Carrera EF, et al. Escolioses idiopáticas. In: Sociedade Brasileira de Ortopedia e Traumatologia. Ortopedia pediátrica. Rio de Janeiro: Revinter; 2004.115-30.

3. Soucacos PN, Zacharis K, Gelalis J, Soultanis K, Kalos N, Beris A, Xenakis T, Johnson EO Assessment of curve progression in idiopathic scoliosis. Eur Spine J 1998.7(4):270-7.

4. Bunnell, W. P.:The natural history of idiopathic scoliosis. Clin. Orthop, 229:20-5, 1988.

5. Díaz J J, Schröter CG, Schulz RI . Actualizacion de La evaluacion radiológica dela escoliosis. Revista Chilena de Radiología. Vol 15. No 3, 2009: 141-51.

6. Kim YJ, Lenke LG, Bridwell KH, Cho YS, Riew KD (2004) Free hand pedicle screw placement in the thoracic spine: is it safe? Spine 29:333-42.

7. Belmont PJ Jr, Klemme WR, Dhawan A, Polly DW (2001) In vivo accuracy of thoracic pedicle screws. Spine 26:2340-6.

8. Sarlak AY, Tosun B, Atmaca H, Sarisoy HT, Buluç L. Evaluation of thoracic pedicle screw placement in adolescent idiopatic scoliosis. Eur Spine J. 2009 Dec, 18(12):1892-7

9. Wang B, Lü G, Chen R, Kang Y, Li J, Chen F, Deng Y, Liu W. Accuracy of free-hand thoracic pedicle screw placement in severe idiopathic scoliosis via CT scan. Journal of Central South University. Medical Sciences. 2009 Mar:34(3):226-9.

10. Rezende R, Machado IC, Barbosa DM, Batista PR. Auxilio da tomografia computadorizada no planejamento pré-operatório de pacientes portadores de escoliose idiopática do adolescente. Coluna/Columna [serial on the Internet]. 2010 Mar [cited 2011 Jan 19]; 9(1): 85-9.

11. Hitesh M, Seung W, Suh HR, Song JY. Accuracy of thoracic pedicle screw placement in scoliosis using the ideal pedicle entry point during the freehand technique

12. Dvorak M, MacDonald S, Gurr KR, Bailey SI, Haddad RG: An anatomic, radiographic, and biomechanical assessment of extrapedicular screw fixation in the thoracic spine. Spine 18:1689-94, 1993. Received: 19 December 2007 / Revised: 12 pação talvez possa diminuir ainda mais o índice de complicações relacionados a acurácia do procedimento. Ainda não foram publicados estudos que demonstrem tal correlação estatística.

Com a violação de 32,9\% dos pedículos, em nossa casuística não observou-se pneumotórax, lesão vascular, lesão de víscera abdominal, lesão da duramáter ou alteração neurológica em nenhum dos 8 pacientes. Todos pacientes deambularam entre o $7^{\circ}$ e $10^{\circ}$ dia pós-operatório e receberam alta antes do $12^{\circ}$ pós-operatório com retornos ambulatoriais subsequentes.

\section{CONCLUSÕES}

Os limites de penetração aceitáveis, assim como os métodos de mensuração ainda não foram padronizados para pacientes portadores de deformidades escolióticas, podendo causar vieses na determinação de risco potencial.

A técnica "free-hand" se mostrou segura do ponto de vista clínico, mesmo com a violação dos pedículos em nosso estudo assim como em outros trabalhos analisados.

A análise das tomografias computadorizadas pós-operatórias mostrou-se uma ferramenta importante na avaliação do risco de lesões vasculares, neurológicas e viscerais, ela possibilita que o cirurgião aprimore suas habilidades e melhore sua acurácia. Também mostrou-se fundamental o estudo com T.C. pré-operatória das escolioses o que permitiu um planejamento cirúrgico afim de evitar os pedículos mais alterados, reduzir o mal posicionamento na colocação de parafusos pediculares e evitar assim possíveis complicações.
January 2008 / Accepted: 12 January 2008 / Published online: 21 March 2008 .SpringerVerlag 2008.

13. Vaccaro AR, Rizzolo SJ, Allardyce TJ, Ramsey M, Salvo J, Balderston Ra, Cotler JM (1995) Placement of pedicle screws in the thoracic spine. Part I: morphometric analysis of the thoracic vertebrae. J Bone Joint Surg Am 77:1193-9.

14. Zindrick MR, Wiltse LL, Doornik A, Widell EH, Knight GW, Patwardhan AG, Thomas JC Rothman SL, Fields BT (1987) Analysis of the morphometric characteristics of the thoracic and lumbar pedicles. Spine 12:160-6.

15. Vauzelle $C$, Stagnara $P$, Jouvinroux P. Functional monitoring of spinal cord activity during spinal surgery. Clin Orthop Relat Res.1973.(93):173-8.

16. Nery LS, Halpern R, Nery PC, Nehme KP, Tetelbom SA. Prevalence of scoliosis among school students in a town in southern Brazil. Sao Paulo Med. J. [serial on the Internet]. 2010 [cited 2011 Jan 19] : 128(2): 69-73.

17. Ebraheim NA, Jabaly G, Xu R, Yeasting RA (1997) Anatomic relations of the thoracic pedicle to the adjacent neural structures. Spine 22:1553-6.

18. Polly DW Jr, Potter BK, Kuklo T, Young S, Johnson C, Klemme WR (2004) Volumetric spinal canal intrusion: a comparison between thoracic pedicle screws and thoracic hooks. Spine.29:63-9.

19. Samdani AF, Ranade A, Sciubba DM, Cahill PJ, Antonacci MD, Clements DH, Betz RR. Accuracy of free-hand placement of thoracic pedicle screws in adolescent idiopathic scoliosis: how much of a difference does surgeon experience make? Eur Spine J. 2009 Oct 29 [Epub ahead of print].

20. Piotr H, Tomasz K, Aleksander K, Andrzej S. The use of computer tomography for preoperative planning and outcome assessment in surgical treatment of idiopathic scoliosis with pedicle screw based constructs-case presentation. Ortopedia, traumatologia, rehabilitacja 2009; 11(6):577-85. 